Ultracold neutral plasmas: recent experiments and new prospects

This article has been downloaded from IOPscience. Please scroll down to see the full text article.

2003 J. Phys. A: Math. Gen. 366077

(http://iopscience.iop.org/0305-4470/36/22/333)

View the table of contents for this issue, or go to the journal homepage for more

Download details:

IP Address: 168.7.217.86

The article was downloaded on 26/11/2012 at 20:10

Please note that terms and conditions apply. 


\title{
Ultracold neutral plasmas: recent experiments and new prospects
}

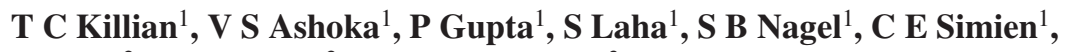 \\ S Kulin ${ }^{2}$, S L Rolston ${ }^{2}$ and S D Bergeson ${ }^{3}$ \\ ${ }^{1}$ Department of Physics and Astronomy and Rice Quantum Institute, Rice University, Houston, \\ TX 77005, USA \\ ${ }^{2}$ National Institute of Standards and Technology, Gaithersburg, MD 20899-8424, USA \\ ${ }^{3}$ Department of Physics and Astronomy, Brigham Young University, Provo, UT 84602-4640, \\ USA
}

Received 22 October 2002

Published 22 May 2003

Online at stacks.iop.org/JPhysA/36/6077

\begin{abstract}
Photoionizing laser-cooled atoms produces ultracold neutral plasmas with initial temperatures of $1-1000 \mathrm{~K}$ and densities as high as $10^{10} \mathrm{~cm}^{-3}$. Applied radio frequency fields can excite plasma oscillations that are used to monitor the expansion of the unconfined plasma. Significant three-body recombination of electrons and ions into Rydberg atoms takes place during the plasma expansion. Previous experiments have been done with xenon, but a new experiment is planned with laser-cooled strontium. The strontium ion has an optically allowed transition at a convenient blue wavelength. This will allow direct imaging of the plasma through fluorescence or absorption, and may enable laser cooling and trapping of the plasma.
\end{abstract}

PACS numbers: 52.55.Dy, 32.80.Pj, 52.25.Ub

\section{Introduction}

Recent experiments conducted at the National Institute of Standards and Technology (NIST) in Gaithersburg opened a new regime of ultracold neutral plasmas with temperatures as low as $1 \mathrm{~K}$. Studies of the methods and conditions for forming the plasma [1], excitation and detection of plasma oscillations [2], dynamics of the plasma expansion [2] and collisional recombination into Rydberg atomic states [3] demonstrated that ultracold neutral plasmas provide a powerful and flexible environment in which to test our fundamental understanding of plasma physics. In related experiments, researchers at the University of Virginia and Laboratoire Aimé Cotton, Orsay, France studied the spontaneous evolution of a dense, cold cloud of Rydberg atoms into a plasma [4]. In this paper, we will review the NIST experiments and then describe future possibilities arising from laser manipulation of the ions after plasma formation. 
The recipe for an ultracold neutral plasma starts with laser-cooled and trapped neutral atoms [5]. Cold atoms are produced and trapped using forces generated by scattering photons from properly arranged laser beams, and many different elements can be used. Alkali atoms, alkaline-earth atoms and metastable noble gas atom are the most common choices. The main requirement is an electric-dipole allowed transition at a convenient laser wavelength. Depending upon the element chosen, up to $10^{10}$ atoms can be trapped. The density can be as high as $10^{12} \mathrm{~cm}^{-3}$, and the temperature can be in the microkelvin to millikelvin range.

To produce the ultracold plasmas, the cold atoms are photoionized barely above threshold with a narrow-bandwidth pulsed laser. The kinetic energy of the original neutral atoms is negligible, so the energetics of the plasma is entirely determined by the photoionization process. Because of the small electron-ion mass ratio, the electrons have an initial kinetic energy $\left(E_{e}\right)$ approximately equal to the difference between the photon energy and the ionization potential. $E_{e} / k_{B}$, where $k_{B}$ is the Boltzmann constant, can be as low as the bandwidth of the ionizing laser, which is $\sim 100 \mathrm{mK}$ with standard pulsed dye lasers. Most studies so far have dealt with $E_{e} / k_{B}$ between 1 and $1000 \mathrm{~K}$. The initial kinetic energy for the ions is in the microkelvin to millikelvin range. Ultracold neutral plasma experiments have so far used xenon [1-3], rubidium [4] and cesium [4].

\section{Review of the experiments with xenon ultracold neutral plasmas}

In the NIST experiments, a few million metastable xenon atoms were cooled to approximately $10 \mu \mathrm{K}$. The peak density was about $2 \times 10^{10} \mathrm{~cm}^{-3}$ and the spatial distribution of the cloud was $n=n_{o} \exp \left(-r^{2} / 2 \sigma^{2}\right)$ with $\sigma \approx 200 \mu \mathrm{m}$. More information on laser cooling and trapping of metastable xenon can be found in [6].

The first experiments studied the dynamics of the formation of the plasma [1]. Immediately after photoionization, the charge distribution is everywhere neutral. Due to the kinetic energy of the electrons, the electron cloud expands, but on this timescale the ions are essentially immobile. The resulting local charge imbalance creates an internal electric field that produces a Coulomb potential energy well for electrons. If the well never becomes deeper than $E_{e}$, all the electrons escape. If enough atoms are photoionized, however, only an outer shell of electrons escapes, and the well becomes deep enough to trap the rest. After the untrapped fraction has escaped, the cloud as a whole is no longer strictly neutral. Simulations show that electrons escape most easily from the edges of the spatial distribution, however, and the centre of the cloud is well described as a neutral plasma.

The number of atoms ionized $\left(N_{i}\right)$, and thus the density of the plasma $(n)$, is controlled by varying the energy of the photoionizing laser pulse. Lower $E_{e}$ and higher $N_{i}$ lead to a greater fraction of the initially created electrons being trapped. For the coldest and densest conditions, over $90 \%$ of the electrons are confined.

For a given $E_{e}$ there is a threshold number of positive ions required for trapping electrons (see figure 1). At the trapping threshold, after all the electrons have left, the potential well depth equals the initial kinetic energy of the electrons. From this relation, one can calculate the number of positive ions at the threshold, $N^{*}=\Delta E / U_{0}$. Here, $U_{0}=\sqrt{2 / \pi} e^{2} / 4 \pi \varepsilon_{0} \sigma$, where $e$ is the elementary charge and $\epsilon_{0}$ is the electric permittivity of vacuum. Equivalently, trapping occurs when the Debye screening length, $\lambda_{D}=\sqrt{\epsilon_{0} k_{B} T_{e} / e^{2} n}$, becomes less than the size of the sample $\sigma$. The electron temperature, $T_{e}$, is approximately equal to $E_{e} / k_{B} \cdot{ }^{4}$ An ionized gas is normally not considered a plasma unless the Debye length is smaller than the size of

\footnotetext{
4 As discussed later in the paper, theoretical results indicate that when the system's initial density and kinetic energy correspond to the region of strong coupling for a single-component system in thermal equilibrium, the charged particles heat rapidly as potential energy associated with disorder is converted into kinetic energy.
} 


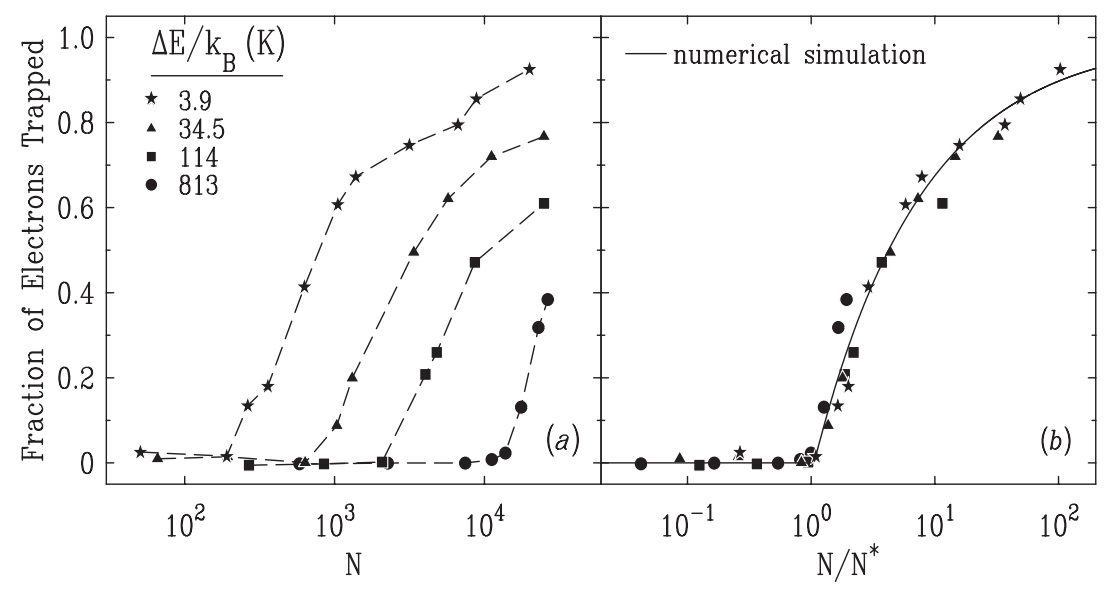

Figure 1. (From [1]) (a) The fraction of electrons trapped is plotted versus the number of photoions created. Each curve corresponds to a different ionizing laser photon energy. The corresponding initial energies of the electrons are displayed in the legend. As the energy increases, more positive ions are required to trap electrons. (b) Same as (a) but the number of photoions is scaled by $N^{*}$. The threshold for trapping is given by $N=N^{*}$. The line is the result of a numerical simulation. There is a scale uncertainty of about $10 \%$ in determining the fraction of electrons trapped.

the system [7], so the threshold for electron trapping is also the threshold for the formation of a plasma. In the xenon experiment, the Debye length can be as low as $500 \mathrm{~nm}$, while the size of the sample is $\sigma \approx 200 \mu \mathrm{m}$. The condition $\lambda_{D}<\sigma$ for creating a plasma is thus easily fulfilled. Electron trapping can also be interpreted as ambipolar diffusion; electrical attraction retards the otherwise rapid outward diffusion of the light electrons.

Figure 2(a) shows electron signals from an ultracold neutral plasma created by photoionization at time $t=0$. A small dc field (about $1 \mathrm{mV} \mathrm{cm}^{-1}$ ) directs free electrons to a single-channel electron multiplier for detection. The first peak at about $1 \mu$ s represents electrons that leave the sample and create the charge imbalance and Coulomb potential well. On a longer timescale, the plasma expands and the depth of the Coulomb well decreases, allowing the remaining electrons to leave the trap. This produces the broad peak at $\sim 25 \mu \mathrm{s}$. Colder and denser plasmas survive for as long as $300 \mu$ s before all the electrons escape.

It is possible to perform experiments on the system during the expansion. In [2], plasma oscillations were excited during this time interval by applying a radio frequency (rf) electric field to the plasma. The oscillations were used to map the plasma density distribution and reveal the particle dynamics and energy flow during the expansion of the ionized gas.

In the absence of a magnetic field, the frequency of plasma oscillations is given by $f_{e}=(1 / 2 \pi) \sqrt{e^{2} n_{e} / \epsilon_{0} m_{e}}$ [8], where $n_{e}$ is the electron density and $m_{e}$ is the electron mass. This expression is valid in a homogeneous medium and for excitations localized in regions of constant density in a spherically symmetric plasma. Analysis of the data assumed excitation of localized modes during the experiment ${ }^{5}$. Plasma oscillations with frequencies from 1 to $250 \mathrm{MHz}$ were observed, corresponding to resonant electron densities between $1 \times 10^{4} \mathrm{~cm}^{-3}$ and $8 \times 10^{8} \mathrm{~cm}^{-3}$.

The applied rf field efficiently excites plasma oscillations and pumps energy into the electron gas when the frequency is resonant with the average density in the plasma $(\bar{n})$.

5 A detailed analysis of the mode structure of plasma oscillations [9] indicates that the finite size of the system shifts the frequency by about a factor of two. This correction has not been included in the analysis. 

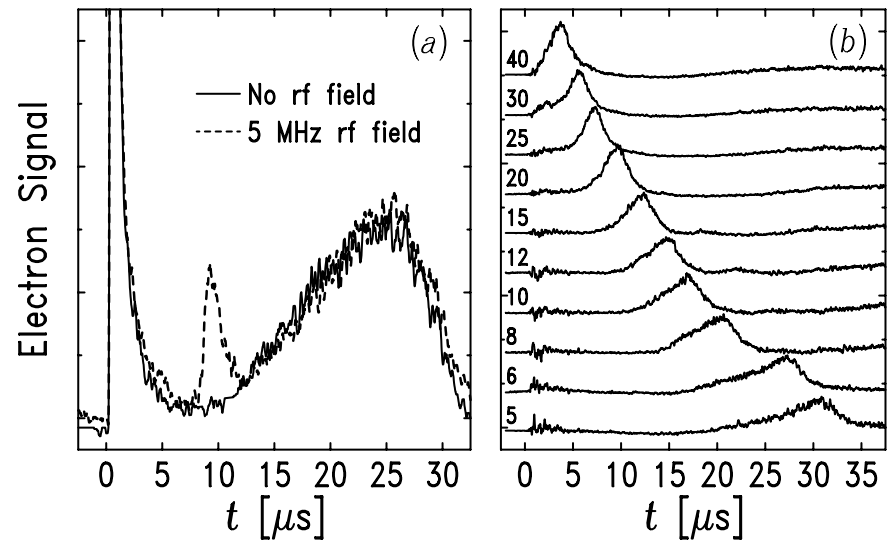

Figure 2. (From [2]) Electron signals from ultracold plasmas created by photoionization at $t=0$. (a) $3 \times 10^{4}$ atoms are photoionized and $E_{e} / k_{B}=540 \mathrm{~K}$. If an rf field is applied during the expansion, resonant excitation of plasma oscillations produces an extra peak on the electron signal. (b) $8 \times 10^{4}$ atoms are photoionized and $E_{e} / k_{B}=26 \mathrm{~K}$. For each trace, the rf frequency in $\mathrm{MHz}$ is indicated, and the nonresonant response has been subtracted. The signals have been offset for clarity. The resonant response arrives later for lower frequency, reflecting expansion of the plasma. For $40 \mathrm{MHz}$, the resonant density is $2.0 \times 10^{7} \mathrm{~cm}^{-3}$; and for $5 \mathrm{MHz}$, it is $3.1 \times 10^{5} \mathrm{~cm}^{-3}$.

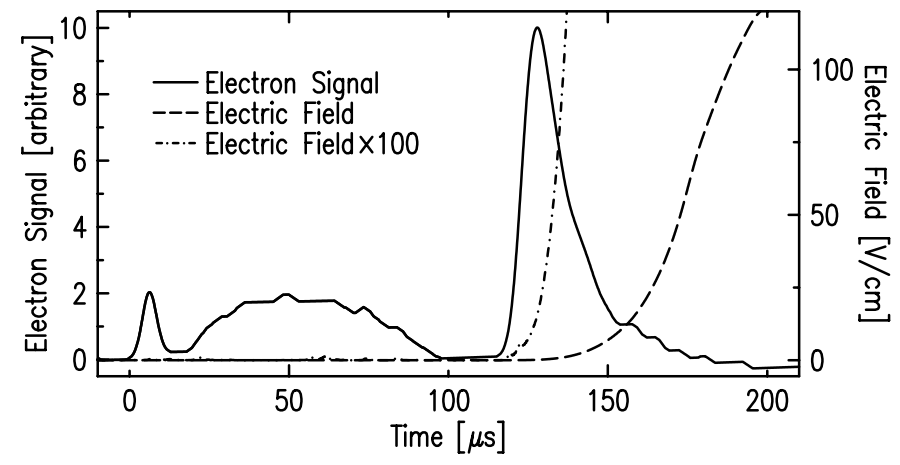

Figure 3. (From [3]) Electron signal from a plasma created by photoionizing $10^{5}$ atoms at $t=0$, with $E_{e} / k_{B}=206 \mathrm{~K}$. The first and second features represent free electrons escaping from the plasma. The third feature arises from ionization of Rydberg atoms. A $5 \mathrm{mV} \mathrm{cm}^{-1}$ field is present before the large-field ramp commences at about $120 \mu \mathrm{s}$, and the collection and detection efficiency for the first and second features is approximately $10 \%$ of the efficiency for electrons from Rydberg atoms.

Collisions redistribute this energy and heat the electrons. This increases the evaporation rate of electrons out of the Coulomb well, which produces the plasma oscillation response on the electron signal (figure 2(a)). The resonant response arrives later for lower frequency (figure $2(b)$ ) as expected because $\bar{n}$ decreases in time. With some analysis, such data implies that after a few $\mu$ s the plasma expands with a constant velocity. A hydrodynamic model developed in [2] shows that the pressure of the electron gas drives the expansion, and the expansion velocity is a sensitive probe of the electron thermal energy at early times.

Recombination into Rydberg atoms in an ultracold neutral plasma was studied in [3]. At temperatures ranging from $1-1000 \mathrm{~K}$, and densities from $10^{5}-10^{10} \mathrm{~cm}^{-3}$, up to $20 \%$ of the initially free charges recombine on a timescale of $100 \mu \mathrm{s}$. Figure 3 is an electron signal 

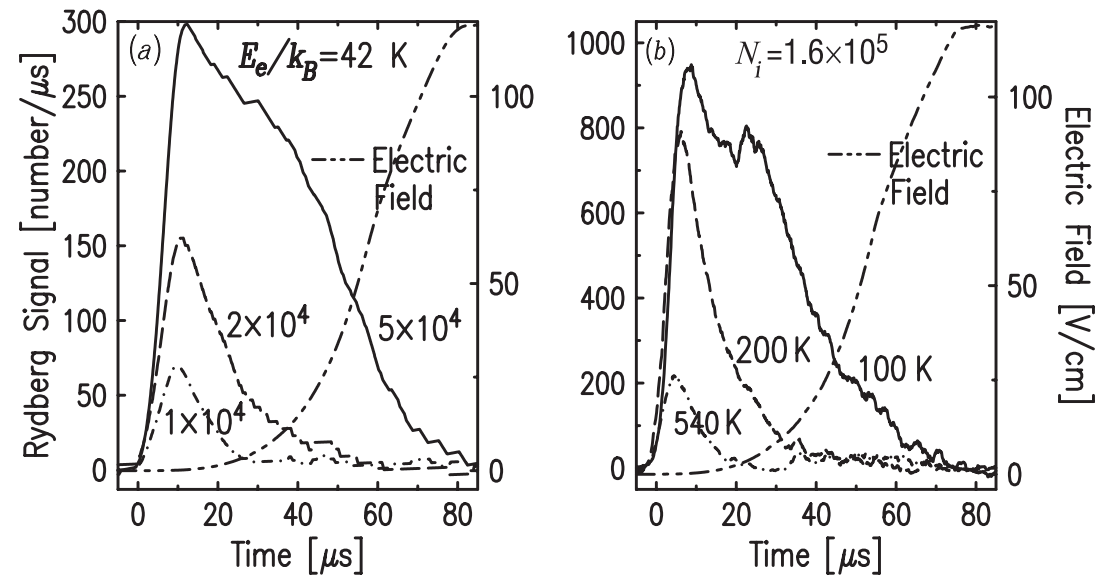

Figure 4. (From [3]) Rydberg ionization signals for various plasma conditions approximately $100 \mu \mathrm{s}$ after photoionization. The time origin is the start of the electric field ramp. (a) Constant $E_{e} / k_{B}=42 \mathrm{~K} . N_{i}$ is indicated near each curve. (b) Constant $N_{i}=1.6 \times 10^{5} . E_{e} / k_{B}$ is indicated near each curve.

from an ultracold neutral plasma that shows the formation of Rydberg atoms. The plasma is formed as described above. After the plasma has expanded so that the ions no longer form a Coulomb well and all free electrons have escaped, the electric field is increased to $120 \mathrm{~V} \mathrm{~cm}^{-1}$ in $\sim 100 \mu$ s. This field can ionize Rydberg atoms bound by as much as $70 \mathrm{~K}$, corresponding to a principle quantum number of about $p=47$. The number of Rydberg atoms formed is inferred from the number of electrons reaching the detector, and the distribution of Rydberg atoms as a function of $p$ is constructed from the fields at which the atoms ionize.

Figure 4 shows typical Rydberg atom data. As the number of ions created $\left(N_{i}\right)$ increases, or $E_{e}$ decreases, a greater fraction of charges recombine and the Rydberg atom distribution shifts towards more deeply bound levels. The integral of each curve yields the total number of Rydberg atoms formed. The expected rates for radiative recombination or dielectronic recombination are many orders of magnitude too low to account for the observed Rydberg atom formation. Three-body recombination (TBR), however, is very fast at ultracold temperatures.

Several theory papers recently addressed the recombination behaviour [10-13]. Full simulations that take into account the collisional cascade of Rydberg atoms to more tightly bound levels qualitatively agree with the observations and confirm that TBR is the dominant mechanism.

These papers, along with [14], also addressed the electron temperature in the system. This was motivated in part by the possibility that these plasmas could be strongly-coupled ${ }^{6}$ due to the low initial kinetic energies and reasonably high densities. The conclusions were that electrons in ultracold neutral plasmas heat to $\Gamma_{e} \lesssim 1$ within a few plasma oscillation periods. Release of energy during TBR, initial random disorder in the system and continuum lowering all played a role in the heating, and it appears that in its present form the experiment will not produce strongly coupled electrons. However, Murillo [15] found that in the current experiment, the ions reach equilibrium in a moderately-coupled liquid state.

\footnotetext{
6 Charged particles in a plasma are strongly coupled when their thermal energy is less than the Coulomb interaction energy between nearest neighbours. The situation is characterized quantitatively by the Coulomb coupling parameter, $\Gamma_{x}=\left(Z_{x}^{2} e^{2} / 4 \pi \varepsilon_{0} a_{x}\right) / k_{B} T_{x}$, where $Z_{x}$ is the charge of species $x, a_{x}=\left(4 \pi n_{x} / 3\right)^{-1 / 3}$ is the Wigner-Seitz radius for density $n_{x}$, and $T_{x}$ is the temperature. In a one-component strongly coupled plasma, $\Gamma>1$ for only one species. In a two-component strongly coupled system, $\Gamma>1$ for positive and negative charges.
} 


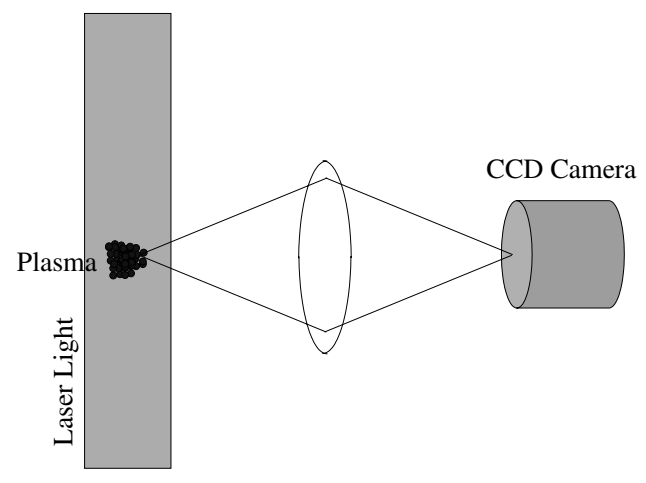

Figure 5. Imaging the plasma ions through resonant light scattering.

\section{Prospects for optically probing a strontium ultracold neutral plasma}

The xenon experiments have demonstrated the fascinating behaviour of ultracold neutral plasmas, but a great present need is for improved diagnostics. For example, if one could resolve variations in the local density, it would be possible to study structural features such as ion acoustic waves or ion-ion spatial correlations. The plasma oscillations that were previously used as a probe were only sensitive to an average density of the plasma.

The ability to directly image the ion density profile through light scattering (figure 5) or absorption imaging would allow simple monitoring of the plasma density as a function of time. The plasma is optically thick ${ }^{7}$ during much of its evolution, which means that optical imaging is straightforward in principle. Such imaging was impossible in previous studies because xenon ions, like most singly charged ions, have principal electronic transitions in the deep ultraviolet—outside the regime of simple lasers and imaging systems. A new apparatus, which is nearly completed, will create ultracold neutral plasmas with atomic strontium and image the plasma with the ion's strong electric dipole transition at $422 \mathrm{~nm}$. Lasers and standard CCD cameras are available at this wavelength. This imaging will be similar to diagnostics used to study clouds of $\mathrm{Be}^{+}$ions in a Penning trap [16].

The ability to image the plasma will allow the study of ion acoustic waves, because they should be directly visible in the ion density profile. One can examine the mode structure of waves in a plasma with a Gaussian density profile. Also, the group velocity of these waves is given by $v=\sqrt{k_{B} T_{e} / m_{i}}$ where $k_{B}$ is the Boltzmann constant, $T_{e}$ is the electron temperature and $m_{i}$ is the ion mass. The group velocity thus could provide a sensitive temperature diagnostic.

It is likely that ion acoustic waves are excited during the formation of the plasma because of the dynamic escape of electrons from the edges. Alternatively, more intense ion acoustic waves could be formed by creating a sharp ion density gradient with a tailored photoionization laser profile.

\section{Laser cooling and trapping the plasma}

Because strontium ions scatter blue light so efficiently, it may be possible to use laser light to cool and trap the ions in a strontium ultracold neutral plasma, just as lasers are used to cool

${ }^{7}$ For optically thick plasmas $n \sigma_{\lambda} d>1$, where $n$ is the plasma density, $\sigma_{\lambda}$ is the ion's photon absorption cross section and $d$ is the plasma diameter. 
and trap neutral atoms. Confining the ions would also confine the electrons through Coulomb attraction. This would be an entirely new method of plasma confinement and may also lead to drastically lower plasma temperatures and strong coupling for the ions.

An estimate of some critical parameters implies that realizing this goal is challenging, but feasible. A magneto-optical trap [17] for atoms or ions has a maximum capture velocity given by $v_{c}=\sqrt{2 F_{\max } r / m}$, where $r$ is the radius of the trapping region where the particles interact with the laser beams, $m$ is the particle mass, and $F_{\max }=\hbar k \gamma / 2$ is the maximum slowing laser force possible. The variable $\gamma$ is the linewidth of the electronic transition used for cooling, $k$ is the laser wavevector and $\hbar$ is Planck's constant divided by $2 \pi$. The maximum force is the momentum imparted during each photon scattering event $(\hbar k)$ times the maximum scattering rate $(\gamma / 2)$. For $\mathrm{Sr}^{+}, \gamma=2 \pi \times 21.5 \times 10^{6} \mathrm{rad} \mathrm{s}^{-1}, k=2 \pi /(422 \mathrm{~nm})$, and using a laser beam interaction radius of $5 \mathrm{~mm}, v_{\mathrm{c}}=85 \mathrm{~m} \mathrm{~s}^{-1}$, which easily exceeds the minimum plasma expansion velocities of $30-40 \mathrm{~m} \mathrm{~s}^{-1}$ observed in [2]. The thermal velocity for ions is much lower than this because the ion-electron thermalization time is very long.

The dominant force behind the plasma expansion arises from the electron pressure [2], and has a typical magnitude of $k_{B} T_{e} / \sigma$, where $\sigma$ is the characteristic radius of the Gaussian plasma density profile. This equals $F_{\max }$ for $10 \mathrm{~K}$ electrons for plasma sizes of $1 \mathrm{~mm}$. Hotter electrons would lead to larger $\sigma$ at which the plasma could be contained.

The lasers will cool the ions in addition to confining them. The velocity-dependent term in the light force, which produces the cooling, can be approximated as $\mathbf{F}=-\beta \mathbf{v}$, where $\beta$ depends upon the laser intensity and detuning from the ion resonance. The kinetic energy damps according to $\mathrm{d} E / \mathrm{d} t=-2 \beta E / m_{i}$, where $m_{i}$ is the ion mass. A simple threedimensional model [18] yields a maximum damping rate $2 \beta_{\max } / m_{i}=\hbar k^{2} /\left(6 m_{i}\right)$. For $\mathrm{Sr}^{+}$, this is equal to $3 \times 10^{4} \mathrm{~s}^{-1}$. The ions will be immersed in a background of hotter electrons, so the cooling will have to counteract heating due to electron-ion equilibration. These issues were discussed in [13], and the electron-ion heating rate was given as

$$
\left(\frac{\mathrm{d} E}{\mathrm{~d} t}\right)_{\text {heat }}=\sqrt{32 \pi} \frac{e^{4} n}{\sqrt{m_{e} k_{B} T_{e}}} \frac{m_{e}}{m_{i}} \ln \left(\frac{\sqrt{3}}{\Gamma_{e}^{3 / 2}}\right)
$$

where $m_{i}$ and $m_{e}$ are the ion and electron masses, $n$ is the plasma density, $\Gamma_{e}$ is the electron Coulomb coupling parameter. For reasonable experimental conditions, $T_{e}=10 \mathrm{~K}$ and $n=10^{8} \mathrm{~cm}^{-3}$, the equilibrium ion temperature is approximately $50 \mathrm{~m} \mathrm{~K}$ and the ion Coulomb coupling parameter is 20 , easily crossing into the strongly-coupled regime. Numerical simulations described in [13] also suggest that the creation of strongly coupled ions is possible with this scheme. In the absence of laser cooling, the ions eventually come into equilibrium with the electrons in a disordered, weakly coupled state.

Many issues need to be addressed experimentally and theoretically, such as the influence of recombination, and loss of electrons from the edge of the plasma, but the development of a completely new mode of plasma confinement is an exciting goal.

\section{Current status of strontium experiments}

We can now laser cool and trap neutral strontium at densities of about $10^{11} \mathrm{~cm}^{-3}$, and we should be able to increase this by another two orders of magnitude. This will allow us to create ultracold plasmas that are about 100 times denser than in the xenon experiments. The photoionization laser is in place, as demonstrated by the photoionization spectrum shown in figure 6. Approximately $1 \mathrm{~mJ}$ per pulse in an area of $1 \mathrm{~cm}^{2}$ ionizes a few per cent of the atoms across most of the spectrum, and at the peak of an autoionizing resonance in the continuum at $405 \mathrm{~nm}$ [19], nearly $20 \%$ of the atoms are ionized. With improvements in power and mode 


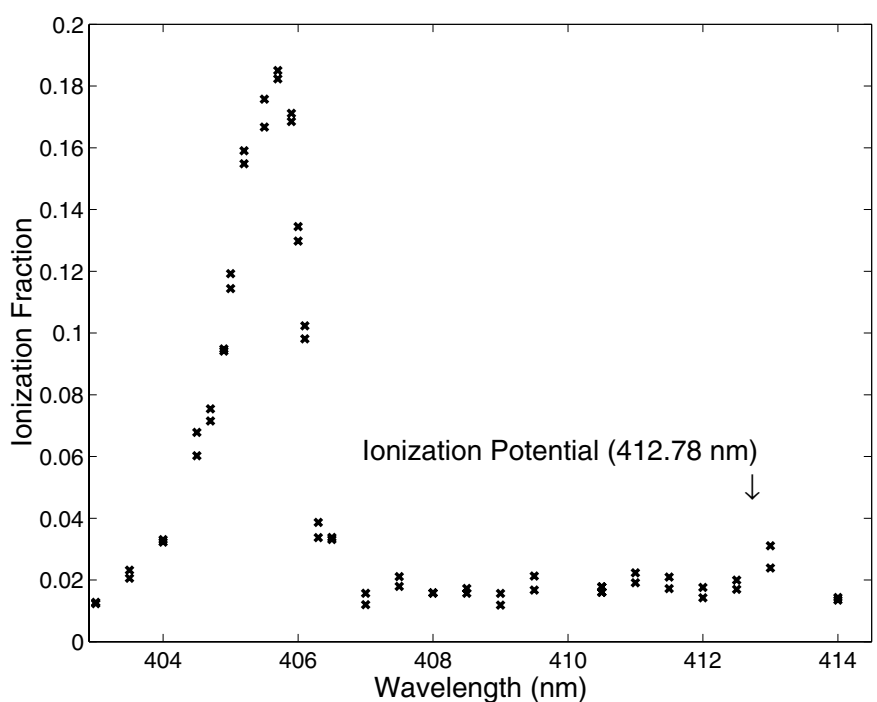

Figure 6. Ultracold strontium atoms held in a magneto-optical trap are ionized with a pulsed dye laser operating at 403-414 nm. Near the peak of an autoionizing resonance at $405 \mathrm{~nm}$, nearly $20 \%$ of the atoms are ionized, which creates ultracold plasmas with densities of about $10^{9} \mathrm{~cm}^{-3}$. Atoms are excited to the continuum from the upper state of the transition used for laser cooling, which is automatically populated by the lasers that cool and trap the neutral atoms.

quality, we expect to ionize at least $50 \%$ of the atoms across the entire spectrum. The last requirement for optical studies of ultracold neutral plasmas is the laser for imaging strontium ions at $422 \mathrm{~nm}$, and construction is proceeding well.

Similar experiments are planned at Brigham Young University, utilizing laser-cooled calcium, which has an internal electronic structure that is similar to strontium's. Ultracold plasma work also continues at NIST, the University of Virginia, the University of Connecticut and the University of Michigan. The future should bring many more exciting results in this new field.

\section{Acknowledgments}

This work was supported by the Department of Energy Office of Fusion Energy Sciences, Office for Naval Research, Research Corporation and Sloan Foundation.

\section{References}

[1] Killian T C, Kulin S, Bergeson S D, Orozco L A, Orzel C and Rolston S L 1999 Phys. Rev. Lett. 834776

[2] Kulin S, Killian T C, Bergeson S D and Rolston S L 2000 Phys. Rev. Lett. 85318

[3] Killian T C, Lim M, Kulin S, Bergeson S D and Rolston S L 2001 Phys. Rev. Lett. 863759

[4] Robinson M P, Laburthe Tolra B, Noel M W, Gallagher T F and Pillet P 2000 Phys. Rev. Lett. 854466

[5] Metcalf H J and van der Straten P 1999 Laser Cooling and Trapping (New York: Springer)

[6] Walhout M, Megens H J L, Witte A and Rolston S L 1993 Phys. Rev. A 48 R879

[7] Chen F F 1974 Introduction to Plasma Physics (New York: Plenum)

[8] Tonks L and Langmuir I 1929 Phys. Rev. 33195

[9] Bergeson S D and Spencer R L 2002 Preprint physics/0204084

[10] Mazevet S, Collins L A and Kress J D 2002 Phys. Rev. Lett. 0255001

[11] Robicheaux F and Hanson J D 2002 Phys. Rev. Lett. 8855002 
[12] Kuzmin S G and O’Neil T M 2002 Phys. Rev. Lett. 8865003

[13] Kuzmin S G and O'Neil T M 2002 Phys. Plasmas 93742

[14] Tkachev A N and Yavlenko S I 2000 Quantum Electron. 301077

[15] Murillo M S 2001 Phys. Rev. Lett. 87 115003-1

[16] Bollinger J J, Mitchell T B, Huang X-P, Itano W M, Tan J N, Jelenković B M and Wineland D J 2000 Phys. Plasmas 77

[17] Raab E L, Prentiss M, Cable A, Chu S and Pritchard D E 1987 Phys. Rev. Lett. 592631

[18] Phillips W D 1992 Proc. International School of Physics 'Enrico Fermi'; Course 118, 'Laser Manipulation of Atoms and Ions: Varenna on Lake Como (Villa Monastero, 9-19 July 1991)’ ed E Arimondo, W Phillips and F Strumia (Amsterdam/New York: North-Holland) pp 289-343

[19] Mende W, Bartschat K and Koch M 1995 J. Phys. B: At. Mol. Opt. Phys. 282385 